\title{
South polar features on Venus similar to those near the north pole
}

G. Piccioni ${ }^{1}$, P. Drossart ${ }^{2}$, A. Sanchez-Lavega ${ }^{3}$, R. Hueso ${ }^{3}$, F. Taylor ${ }^{4}$, C. Wilson ${ }^{4}$, D. Grassi $^{2}$, L. Zasova ${ }^{5}$, M. Moriconi ${ }^{6}$, A. Adriani ${ }^{7}$, S. Lebonnois ${ }^{8}$, A. Coradini ${ }^{7}$, B.

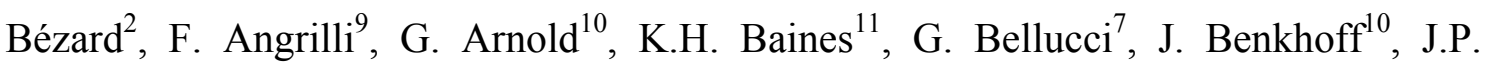
Bibring $^{12}$, A. Blanco ${ }^{13}$, M. I. Blecka ${ }^{14}$, R.W. Carlson ${ }^{11}$, A. Di Lellis ${ }^{15}$, T. Encrenaz ${ }^{2}$, S.

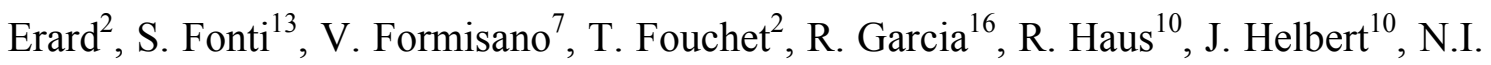
Ignatiev $^{5}$, P. Irwin ${ }^{4}$, Y. Langevin ${ }^{12}$, M.A. Lopez-Valverde ${ }^{17}$, D. Luz ${ }^{2}$, L. Marinangeli ${ }^{18}$, V. Orofino ${ }^{13}$, A.V. Rodin ${ }^{5}$, M. C. Roos-Serote ${ }^{19}$, B. Saggin ${ }^{20}$, D. M. Stam ${ }^{21}$, D. Titov ${ }^{22}$, G. Visconti ${ }^{23}$, M. Zambelli ${ }^{1} \&$ the VIRTIS-Venus Express Technical Team ${ }^{\mathrm{i}}$

1 INAF-IASF (Istituto di Astrofisica Spaziale e Fisica Cosmica), via del fosso del Cavaliere 100, 00133 Rome (Italy)

2 LESIA, Observatoire de Paris, CNRS, UPMC, Université Paris-Diderot, 5 place Jules Janssen, 92195 Meudon, France

3 Dpto. Física Aplicada I Escuela Superior de Ingenieros Universidad del Pais Vasco Alda. Urquijo s/n 48013, BILBAO (Spain)

4 University of Oxford, Department of Physics Atmospheric, Oceanic and Planetary Physics Clarendon Laboratory, Parks Road, Oxford OX1 3PU (United Kingdom)

5 Space Research Institute of Russian Academy of Sciences (IKI), Profsojuznaja 84/32, 117997 Moscow, (Russia)

\footnotetext{
${ }^{\mathrm{i}}$ The VIRTIS-Venus Express Technical Team is listed at the end of this paper
} 
6 CNR-ISAC (Istituto di Scienze dell'Atmosfera e del Clima), via del fosso del cavaliere 100, 00133 Rome (Italy)

7 INAF-IFSI (Istituto di Fisica dello Spazio Interplanetario), via del fosso del cavaliere 100, 00133 Rome (Italy)

8 Laboratoire de Meteorologie Dynamique, Jussieu, Box 9975252 PARIS cedex 05 (France)

9 CISAS Università di Padova, via Venezia 1, 35131 Padova (Italy)

${ }^{10}$ German Aerospace Center (DLR) Institute of Planetary Exploration Planetary Physics, Berlin-Adlershof Rutherfordstrasse 2, 12489 Berlin (Germany)

11 Jet Propulsion Laboratory, MS 183-601 Pasadena CA 91011 (United States)

12 Institut d'Astrophysique Spatiale, Bâtiment 120 Université Paris-Sud, 91405 Orsay Cedex (France)

${ }^{13}$ Università degli Studi di Lecce Dipartimento di Fisica, Via Arnesano, 73100 Lecce (Italy)

14 Space Research Centre of Polish Academy of Science, Bartycka 18A, 00-716 Warszawa, (Poland)

15 AMDLSPACE s.r.l., Via Giovanni Angelini 3300149 Rome (Italy)

${ }^{16}$ Département des Études Spatiales Institut de Physique du Globe de Paris 4, Avenue de Neptune F-94107 Saint Maur des Fossés cedex (France)

17 Instituto de Astrofísica de Andalucía (CSIC), Camino Bajo de Huétor, 24 Apartado 3004, 18080 Granada (Spain)

18 International Research School of Planetary Sciences Dipartimento di Scienze Universita' d'Annunzio, Viale Pindaro 42, 65127 Pescara (Italy)

${ }^{19}$ Observatorio Astronomico de Lisboa Centro de Astronomia e Astrofisica da Universidade de Lisboa, Tapada da Ajuda 1349-018, Lisboa (Portugal) 
${ }^{20}$ Politecnico di Milano, Polo di Lecco, Via Marco D'Oggiono 18/A, 23900 Lecco (Italy)

21 Astronomical Institute "Anton Pannekoek" University of Amsterdam, Kruislaan 403 1098 SJ, Amsterdam (The Netherlands)

${ }^{22}$ Max-Planck-Institute for Aeronomy, Max Planck Str. 2, 37191 Katlenburg-Lindau (Germany)

23 Dipartimento di Fisica Università de L'Aquila, via Vetoio Loc. Coppito, 67010 Coppito, L'Aquila (Italy) 
Venus has no seasons, slow rotation and a very massive atmosphere consisting mainly of carbon dioxide with clouds thought to be made primarily of sulphuric acid droplets, all of which make it strikingly different from Earth and Mars. Infrared observations by previous missions to Venus revealed a bright 'dipole' feature surrounded by a cold 'collar' at its north pole ${ }^{1,2,3,4}$. The polar 'dipole' is a double-eye feature at the centre of a vast vortex that rotates around the pole, and is possibly associated with rapid downwelling. The polar cold 'collar' is a wide, shallow river of cold air which circulates around the polar vortex. One outstanding question has been whether the global circulation was symmetric, such that a dipole feature existed at the south pole. Here we report observations of Venus' south polar region, where we have seen clouds with morphology much like those around the north pole, but rotating somewhat faster than the rotation rate reported for the northern dipole. We report that in the south polar region the spectroscopic properties of the clouds are compatible with a sulphuric acid composition. We also report here that the vortex may extend down at least to the lower clouds layers lying at about $50 \mathrm{~km}$ height and perhaps deeper.

We used the Visible and InfraRed Thermal Imaging Spectrometer (VIRTIS) instrument ${ }^{\mathbf{5 , 6}}$ on the Venus Express spacecraft, to observe the south polar region of Venus.

VIRTIS measures radiation intensity at wavelengths between 0.3 and $5 \mu \mathrm{m}$. The wavelengths between 3 and $5 \mu \mathrm{m}$ are sensitive to both temperature and cloud opacity variations. Despite the uncertainties this introduces, extensive radiative transfer modelling indicates that the real temperature profile in the mesosphere $(60-100 \mathrm{~km}) \mathrm{can}$ be retrieved from VIRTIS data with errors within a few K. The observations in Fig. 1 show that the structure of the South pole as seen in the thermal region at about $5 \mu \mathrm{m}$ 
exhibits an inverse "S" shape, indicating a polar dipole having two centres of rotation in counter-clockwise direction, in agreement with the direction of the atmospheric superrotation, surrounded by a cold collar very much like that observed in the North from past missions. Both the dipole and the collar are thus similar to those previously observed at the north pole by Pioneer Venus ${ }^{4}$ but in mirror image (due to the clockwise rotation of the atmosphere at the north pole). The horizontal gradient of temperature is greatest at an altitude of about $60 \mathrm{~km}$ (the lowest layer effectively probed in the thermal region at about $5 \mu \mathrm{m}$ ), with a brightness temperature as high as $250 \mathrm{~K}$ observed inside the dipole in contrast with the temperature as low as $210 \mathrm{~K}$ observed in the cold collar region. This is shown in Fig. 2, where the real retrieved temperatures are also reported. The detailed, double-eyed structure seen in Fig. 1,2 is not always observed; an ovalshaped dipole with less contrast and variable morphology is more frequently observed in the VIRTIS data from the first few months of Venus Express operations.

Images of the dipole taken during the first 45 days at Venus indicate that it rotates with a period of $(-2.48 \pm 0.05)$ days. The negative sign indicates that the dipole rotates in the direction of the solid body of the planet and the superrotating atmosphere, which is retrograde with respect to the rotation of the Earth and most other planets in the solar system. This rotation is somewhat faster than the rotation of the Northern dipole observed from Pioneer Venus in 1979, which exhibited a rotation period varying from 2.79 days to -3.21 days over the 72 days of observation ${ }^{7}$. It could be that the difference is in part due to hemispherical asymmetry; but temporal variability also seems to play an important role. The variability may be caused by external sources - for example solar influences - or may be indicative of dynamical instability in the atmospheric circulation. The VIRTIS observations reported here were taken sporadically during 
spacecraft commissioning, so we cannot yet quantify the variability of the dipole rotation rate. However, further observations will help clarify this question.

The analysis of 3-dimensional atmospheric thermal fields retrieved from the measured radiances indicates that regions of cold atmosphere $(210 \mathrm{~K})$ are associated with the cold collar at the 100 mbar level (about $65 \mathrm{~km}$ altitude). This spatial structure vanishes at higher altitudes $(>75 \mathrm{~km})$ where the layers become more spatially isothermal. Atmospheric vertical temperature gradients between 100 to 50 mbar (about $68 \mathrm{~km}$ altitude) appear moderate inside the " $\mathrm{S}$ " shape, in the range 0 to $-3 \mathrm{~K} / \mathrm{km}$, while strong inversions are observed above the cold collar with values up to $6 \mathrm{~K} / \mathrm{km}$. Another weak maximum in vertical atmospheric temperature profiles is observed around 0.5 mbar (about $90 \mathrm{~km}$ altitude), with a peak temperature ranging from 185 to $195 \mathrm{~K}$ and apparently no clear spatial organization. The coldest side of the cold collar is identified on the morning terminator side similarly to the one observed on the north pole. The centre of the dipole from a thermal point of view, as shown in Fig. 1 is $4^{\circ}$ offset with respect to the geometric South pole, although this displacement evidently varies with time. The structure of the dipole at different cloud heights and wavelengths is shown in the supplementary Fig. 1.

Figure 3 shows the first ever image of the deep atmospheric structure of the vortex in two of the spectral 'window' regions at wavelengths near 1.74 and $2.3 \mu \mathrm{m}$. Radiative transfer models indicate that the radiation at these wavelengths originates about $10-20$ and $20-30 \mathrm{~km}$ above the surface, respectively ${ }^{\mathbf{8}, 9}$. The spatial contrast structure is produced as this radiation passes through the clouds and is attenuated by cloud layers with differing optical depths ${ }^{\mathbf{1 0 , 1 1 , 1 2}}$. It was found earlier from Venera 15 IR spectrometry 
that sulphuric acid is the main component of the clouds in the north polar region ${ }^{\mathbf{1 3}}$. From Mie theory calculations, at $\lambda<1.27 \mu \mathrm{m}$ the single scattering albedo of sulphuric acid particles is $\omega_{0}>0.9999$ against $\omega_{0}=0.988$ at $2.35 \mu \mathrm{m}$. The relative variation of radiance in the near infrared spectral windows as shown in Fig. 3 and supplementary Fig. 1 is compatible with a sulphuric acid composition of the clouds. The increasing thickness of the clouds does not greatly influence the conservative scattering part of the spectrum $(\lambda<1.27 \mu \mathrm{m})$, as may be seen in Fig. 3 and panel i of supplementary Fig. 1. This can be considered as the first indication of the sulphuric acid composition of the clouds in the south polar region.

The polar vortex region is typically very cloudy even though there are occasionally 'holes' where it is possible to probe very deep in altitude at wavelength of $1.74 \mu \mathrm{m}$. The outline of the dipole seen in the thermal region corresponding to the upper clouds layer, overlaps with the pattern seen in the near infrared which is largely modulated by the opacity of the lower and middle clouds layers, but the structure is somewhat different, see Fig. 3." The images probing the deep atmosphere show that the vortex may extend as far as the base of the cloud layer at about $50 \mathrm{~km}$ height and perhaps deeper.

VIRTIS observations usually show a strong correlation between the details observed at about 5.0 and 3.8 microns, suggesting that the radiance at these wavelengths may depend on the thermal emission from clouds at about the same level. In contrast, simultaneous observations at UV and infrared wavelengths taken on orbit 29 (19th May 2006), see supplementary Fig. 1, show a strong anticorrelation of the dark UV cloud features and bright emission regions in the infrared in both the dipole and the spiral arm around it. A possible explanation is that the dark UV features absorb more solar radiation and are actually warmer than the UV bright features, however heating by solar 
radiation is less effective at high latitudes in the polar region. Alternatively, bright regions in the $400 \mathrm{~nm}$ images of the high latitude dayside may also be interpreted as being due to higher clouds located below the thermal inversion layer. Such clouds would have lower temperatures and appear dark in the thermal IR and the effective level from which radiation comes in the UV spectral range would be in this case significantly lower than the previously estimated value around $70 \mathrm{~km}$ height ${ }^{\mathbf{1 4 1 1 5 , 1 6}}$.

Small-scale features in the images taken at 1.74 and $5.05 \mu \mathrm{m}$ during orbit 38 were tracked to derive winds at different vertical depths. The wind velocity measured at 1.74 $\mu \mathrm{m}$ (assumed to originate at $45-50 \mathrm{~km}$ altitude) decreases from $50 \pm 3 \mathrm{~ms}^{-1}$ at latitude $75^{\circ} \mathrm{S}$ to $0 \mathrm{~ms}^{-1}$ at the pole implying a meridional wind shear of $(3.2 \pm 0.2) \times 10^{-5} \mathrm{~s}^{-1}$. This matches the rotation rate of the large-scale dipole feature at this wavelength. Small scale features observed in the dipole at $5.05 \mu \mathrm{m}$ (altitude about $60 \mathrm{~km}$ ) move with velocities that decrease from $35 \pm 5 \mathrm{~ms}^{-1}$ at $75^{\circ} \mathrm{S}$ to $0 \mathrm{~ms}^{-1}$ at the pole, implying a meridional wind shear of $(2.2 \pm 0.3) \times 10^{-5} \mathrm{~s}^{-1}$. Both the upper and lower structures move at the same velocity at latitudes poleward of $-80^{\circ}$. The mean vertical wind shear at the dipole external limit at $-75^{\circ}$ is $(1.5 \pm 0.5) \times 10^{-3} \mathrm{~s}^{-1}$ assuming the 1.74 and $5.05 \mu \mathrm{m}$ images are sensing at the $50 \mathrm{~km}$ and $60 \mathrm{~km}$ altitudes respectively. The interesting time and structure variability of the dipole will be further analysed over the course of the mission. 


\section{References:}

1 Taylor, F.W., McCleese, D.J., and Diner, D.J., "Polar clearing in the Venus clouds observed from the Pioneer Venus Orbiter”. Nature 279, 613-614, (1979).

2 Elson LS, 'Wave Instability in the Polar Region of Venus'. Journal of the Atmospheric Sciences 39, 2356-2362, (1982).

3 Taylor, F.W., “The Venusian Polar Dipole”. In 'Middle Atmosphere of Venus', ed. K. Schaeffer and D Spankuch, Veroffentlichungen des Forchungsberichs Geo- und Kosmoswischenschaften, Academie-Verlag Berlin.18, 93-97, (1990).

4 Taylor, F.W., et al., “Temperature, Cloud Structure, and Dynamics of Venus Middle Atmosphere by Infrared Remote-Sensing from Pioneer Orbiter”. Science 205, 65-67, (1979)

5 Drossart, P., et al., "Scientific goals for the observation of Venus by VIRTIS on ESA/Venus Express mission". Planetary and Space Science, in press.

6 Piccioni, G., et al., "VIRTIS: the Visible and Infrared Thermal Imaging Spectrometer". ESA-SP, in press

7 Schofield, J.T. \& Diner, D.J., "Rotation of Venus Polar Dipole”. Nature 305, 116-119, (1983). 
8 Bezard, B., at al., "The deep atmosphere of Venus revealed by high-resolution nightside spectra”, Nature 345, 508-511, (1990).

9 Pollack, J. B., et al., "Near infrared light from Venus' nightside: A spectroscopic analysis", Icarus 103, 1-42, (1993).

10 Allen, D. A. and Crawford, J. W., "Cloud structure on the dark side of Venus", Nature 307, 222-224, (1984).

11 Kamp, L. W., Taylor F. W., and Calcutt S. B., "Structure of the Venus atmosphere for modelling of night side infrared spectra”, Nature 336, 360-362, (1988).

12 Crisp, D., et al., "The nature of the near-infrared features on the Venus night side", Science 246, 506-509, (1989).

13 Zasova, L. V., et al., "Structure of the Venus atmosphere". Planetary and Space Science 55, (2007), in press

14 Esposito, L.W.; et al., "Chemistry of Lower Atmosphere and Clouds”. In VENUS II: Geology, Geophysics, Atmosphere and Solar Wind Environment, University of Arizona Press, 415-458, (1997).

15 Murray, Bruce C.; et al., 'Venus: Atmospheric Motion and Structure from Mariner 10 Pictures'. Science 183, 1307-1315, (1974). 
16 Suomi VE \& Limaye SS, 'Venus - further evidence of vortex circulation'. Science 201, 1009-1011, (1978).

17 Grinspoon, D.H., et al., "Probing Venus's cloud structure with Galileo NIMS". Planetary And Space Science 41, 515-542, (1993).

18 Carlson, R.W., et al., "Variations In Venus Cloud Particle Properties - A New View Of Venus's Cloud Morphology As Observed By The Galileo Near-Infrared Mapping Spectrometer”. Planetary And Space Science 41, 477-485 , (1993).

Supplementary information is linked to the online version of the paper at www.nature.com/nature.

Acknowledgements We gratefully acknowledge the work of the entire Venus Express team that allowed these data to be obtained. We wish to thank ASI, CNES and the other national space agencies that have supported this research.

Author Contributions G.P. and P.D. have coordinated the work as Principal Investigators of VIRTIS. A.S.L. and R.H. have contributed to the dynamics calculation and to the supplementary figure 1. F.T., C.W. and L.Z. have contributed to the comparison with earlier data and to the finalization of the paper. D.G. has contributed to the temperature retrieval model and calculation, M.M. and A.A. have contributed to the thermal structure. S.L. has contributed to the coordination of the dynamics study. All authors have contributed equally to the planning of the work and data analysis. 
Author information The authors declare no competing financial interests.

Correspondence and requests for materials should be addressed to G.P., (Giuseppe.piccioni@iasf-roma.inaf.it) 
The VIRTIS-Venus Express Technical Team :

Ammannito Eleonora ${ }^{1}$, Barbis Alessandra ${ }^{2}$, Berlin Rainer ${ }^{3}$, Bettanini Carlo ${ }^{4}$, Boccaccini Angelo $^{1}$, Bonnello Guillaume ${ }^{5}$, Bouye Marc $^{6}$, Capaccioni Fabrizio ${ }^{7}$, Cardesin Alejandro $^{7}$, Carraro Francesco ${ }^{8}$, Cherubini Giovanni $^{2}$, Cosi Massimo $^{2}$, Dami Michele $^{2}$, De Nino Maurizio ${ }^{9}$, Del Vento Davide ${ }^{7}$, Di Giampietro Marco $^{2} \quad$, Donati Alessandro ${ }^{2}$, Dupuis Olivier ${ }^{6}$, Espinasse Sylvie $^{8}$, Fabbri Anna $^{2}$, Fave Agnes ${ }^{6}$, Ficai Veltroni Iacopo ${ }^{2}$, Filacchione Gianrico ${ }^{7}$, Garceran Katia ${ }^{6}$, Ghomchi Yamina ${ }^{6}$, Giustini Maurizio ${ }^{2}$, Gondet Brigitte $^{5}$, Hello Yann ${ }^{6}$, Henry Florence ${ }^{6}$, Hofer Stefan ${ }^{10}$, Huntzinger Gerard ${ }^{6}$, Kachlicki Juergen $^{3}$, René Knoll ${ }^{6}$, Kouach Driss ${ }^{6}$, Mazzoni Alessandro ${ }^{2}$, Melchiorri Riccardo ${ }^{6}$, Mondello Giuseppe ${ }^{2}$, Monti Francesco ${ }^{9}$, Neumann Christian ${ }^{10}$, Nuccilli Fabrizio ${ }^{1}$, Parisot Jerome ${ }^{6}$, Pasqui Claudio ${ }^{2}$, Perferi Stefano ${ }^{2}$, Peter Gisbert ${ }^{3}$, Piacentino Alain ${ }^{6}$, Pompei Carlo ${ }^{2}$, Reess Jean-Michel ${ }^{6}$, Rivet Jean-Pierre ${ }^{6}$, Romano Antonio ${ }^{2}$, Russ Natalie $^{3}$, Santoni Massimo ${ }^{2}$, Scarpelli Adelmo ${ }^{2}$, Semery Alain ${ }^{6}$, Soufflot Alain ${ }^{5}$, Stefanovitch Douchane ${ }^{6}$, Suetta Enrico ${ }^{2}$, Tarchi Fabio $^{2}$, Tonetti Nazzareno ${ }^{2}$, Tosi Federico $^{1} \&$ Bernd Ulmer ${ }^{3}$

Affiliations for participants: ${ }^{1}$ INAF-IFSI Rome, ${ }^{2}$ Galileo Avionica Florence, ${ }^{3}$ DLR Berlin, ${ }^{4}$ Università di Padova, ${ }^{5}$ IAS Orsay, ${ }^{6}$ LESIA, Obs. de Paris, ${ }^{7}$ INAF-IASF Rome, ${ }^{8}$ ASI Rome, ${ }^{9}$ Techno System developments Naples, ${ }^{10}$ Kayser Threde Muenchen 


\section{Figures captions}

\section{Figure 1: The Venus southern polar dipole.}

A sequence of four images at a wavelength of $5.05 \mu \mathrm{m}$ acquired during orbit 38 on May

28 2006, time 17:41:30, starting from (a) with a time interval of 1 h. The dipole was clearly seen in these observations with an unprecedented detail from a distance of about $60,000 \mathrm{~km}$ as it rotates around the pole. The images provided by VIRTIS, when used in high spatial resolution mode such as in this figure, have a size of 256 by 256 pixels and each pixel has an instantaneous field of view of $250 \mu \mathrm{rad}$. Major and minor axis dimensions of the dipole are about $2700 \mathrm{~km}$ and $900 \mathrm{~km}$ respectively. Radiative transfer calculation sets the main source of the radiance at this wavelength as coming from a layer at about $60 \mathrm{~km}$ altitude where the maximum contrast is seen for the detailed thermal structure of the dipole. The blue and the green curves in the four panels are the meridians at $330^{\circ}$ and $350^{\circ}$ of longitude respectively. The red circle indicates the south pole while the yellow curve is the parallel at $-70^{\circ}$ of latitude. The cold collar is just beyond this latitude and its temperature is coldest on the right side, where the morning terminator resides. The centre of the dipole in temperature is offset by 4 deg with respect to the south pole in this image. However we expect some variability that can be studied over a long term period during the course of the mission.

\section{Figure 2: The dipole in its highest thermal contrast atmospheric layer.}

The thermal brightness at $5.05 \mu \mathrm{m}$ of the dipole and the cold collar region.

The level curves in the image are the real atmospheric temperatures retrieved from the VIRTIS measured radiances correspondent to a pressure of $200 \mathrm{mbar}$ (about $60 \mathrm{~km}$ ) and they correlate pretty well with the brightness temperatures at this wavelength. The brightness temperature contrast is maximum at this layer and the warmer region 
corresponds to the ellipse containing the dipole where a brightness temperature as high as $250 \mathrm{~K}$ is observed in the centre of rotation on the left side (real retrieved atmospheric temperature being only a few $\mathrm{K}$ less). This part was on the day side at this time and thus it may be warmed by the solar flux. The overall contrast inside the ellipse is not more than $8 \mathrm{~K}$ at $5.05 \mu \mathrm{m}$.

The spatial thermal gradient is steepest from the cold collar to the inner part on the right side of the right hand pole of the dipole where a temperature difference of $15 \mathrm{~K}$ is observed in a distance of the order of $200 \mathrm{~km}$, just outside the edge of the ellipse. The coldest region of the polar collar with temperatures of about $210 \mathrm{~K}$ is registered on the right part, on the morning terminator side. The atmospheric vertical temperature profile in two different regions is shown in the graphs on the right side. The green curve is retrieved in the green spot on the left image, inside the polar collar region. This is also the region where the more prominent thermal inversion is observed in the range from 60 to $70 \mathrm{~km}$ altitude. The red curve is from the red spot inside the dipole where the thermal inversion is much more limited.

The image also reveals filaments in the circular shape following the rotation of the dipole in the outer part of the ellipse, and also a fine detailed structure in the inner part where the two bright features seem to be connected together.

\section{Figure 3: The deepest altitude view of the dipole ever seen.}

Night-side images at $1.74 \mu \mathrm{m}$ (a) and $2.3 \mu \mathrm{m}$ (b) selected from spectral image cube number 4 of orbit 38 . The thermal radiation at these wavelengths mainly originates below the cloud layers, so the structure is produced by spatial variations in the opacity of the clouds. The morphology of the vortex here is remarkable and presents many details. The circular features falling in the region of the cold collar are probably due to 
clouds elongated by the strong winds coming from the superrotation. The same cloud structures are seen in both spectral regions with difference in the contrast of the features.

The reason of the lower signal at $2.3 \mu \mathrm{m}$ can be attributed to the different number density or size of the clouds particles ${ }^{\mathbf{1 7}, 18}$. The single scattering albedo for sulphuric acid mode 2 particles is $\omega_{0}=0.99978$ and 0.988 , respectively, for these windows. In the 1.74 $\mu \mathrm{m}$ wavelength the signal decreases and in the $2.3 \mu \mathrm{m}$ the signal becomes dramatically low, sometimes disappearing altogether when the cloud optical depth increases. This is shown in two spectra in panel $\mathbf{b}$ related to two different regions, bright (green circle and green spectrum) and dark (red circle and red spectrum), see supplementary Fig. 1 for further information. The rising of the continuum radiance on the left side of the spectrum (shorter wavelengths) is due to contamination by scattered light coming from the day side. The behaviour of the windows corresponds to the variation of the cloud optical depth: the intensity does not change significantly in the window with conservative scattering (for wavelengths lower than $1.27 \mu \mathrm{m}$, the absorption optical depth of the cloud deck is less than unity) but changes strongly in the $2.3 \mu \mathrm{m}$ window. The whole of the dipole is not visible at these wavelengths, because the strong reflected solar light from the higher levels on the day side does not allow the detection of the deeper levels of the structure beyond the terminator. The 3D view of the vortex seen in these images is not typical and it may have been enhanced by the relatively high emergence angle of these observations, about $40 \mathrm{deg}$ in the centre and ranging from 20 to $60 \mathrm{deg}$ from the left top to the right bottom corner. 


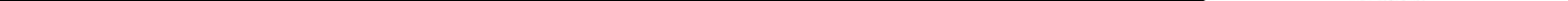



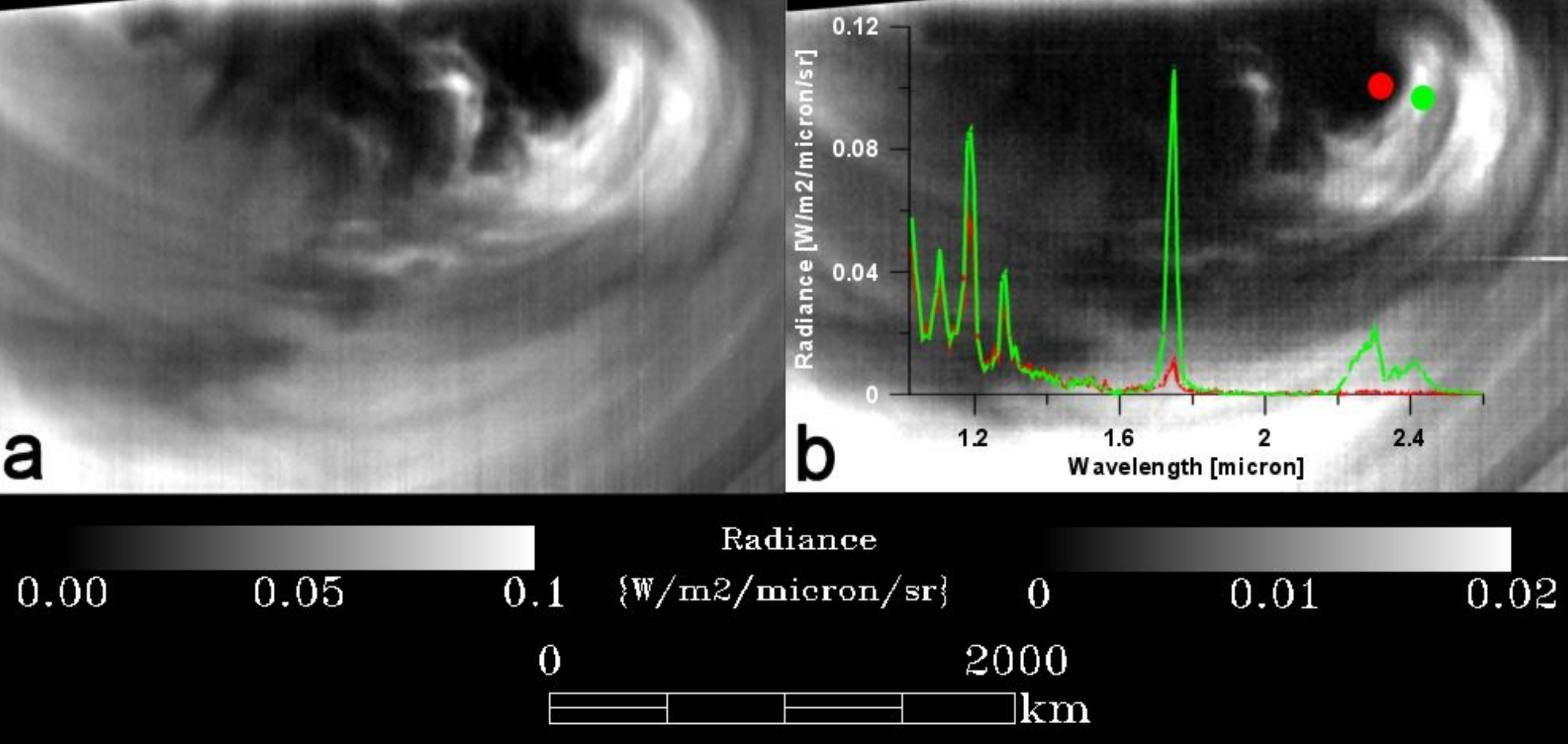